\title{
Visuelle Täuschungen beim Charles-Bonnet-Syndrom
}

\author{
Dr. med. Rolf Erlebacha, Dr. med. Lutz Seebach ${ }^{a}$, PD Dr. med. Esther Bächlia , PD Dr. med. Sarah Martia,b \\ ${ }^{a}$ Departement Medizinische Disziplinen, Medizinische Klinik, Spital Uster; ${ }^{b}$ Neurologische Praxis, Zollikerberg
}

Die Korrespondenzadresse wurde korrigiert.

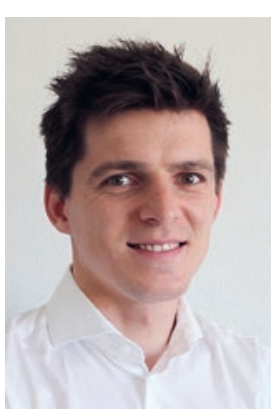

Rolf Erlebach

\section{Hintergrund}

Das Charles-Bonnet-Syndrom umfasst verschiedene visuelle Phänomene, die bei Personen mit stark eingeschränktem Sehvermögen auftreten und die Lebensqualität negativ beeinflussen. Wir berichten über einen 81-jährigen Mann mit vorbestehender Makuladegeneration und neu aufgetretenen visuellen Täuschungen.

\section{Fallbericht}

\section{Anamnese}

Ein 81-jähriger Mann stellte sich mit neuartigen Sehstörungen auf Zuweisung notfallmässig vor. Er beschrieb fünf- bis sechseckige, netzartig verbundene Figuren, die er an verschiedenen Stellen im Gesichtsfeld beider Augen wahrnahm. Die Figuren traten vor allem beim entspannten Blick auf eine weisse Fläche auf und waren schwarz gefärbt. Sie bewegten sich mit dem Blick des Patienten und verschmolzen teilweise mit dem Rest des Bildes (Abb. 1).

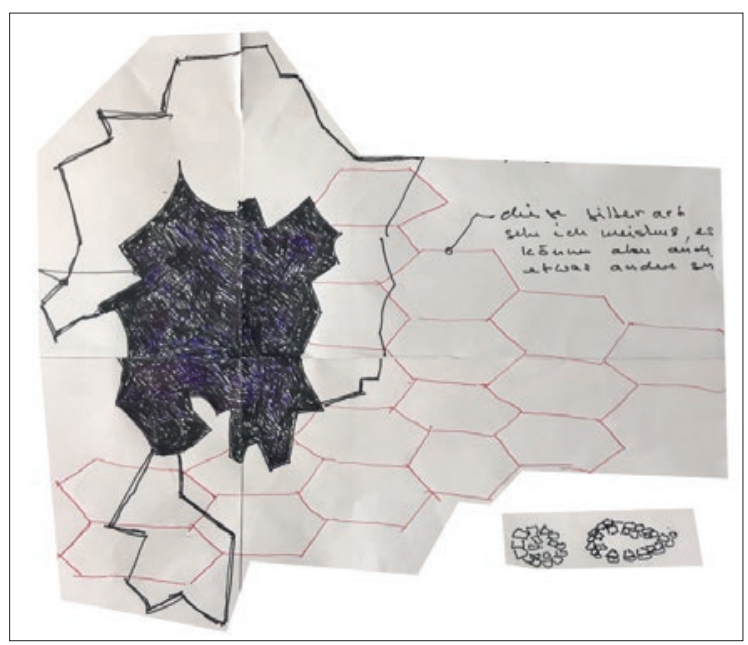

Abbildung 1: Sechseckige, netzartige Muster, die sich auf ein schwarz-weisses Hintergrundbild legen (vom Patienten gezeichnet). Das rote Netz von geometrischen Formen wird vom Patienten schwarz gesehen, was für ihn die Unterscheidung mit dem Hintergrundbild erschwert. Weitere vom Patienten beschriebene und nachgezeichnete zirkuläre Anordnung eckiger Strukturen (unten rechts).
Ausserdem passierte der Patient während des Morgenspazierganges einen blühenden grünen Busch, der sich anschliessend nicht aus dem Blickfeld löste.

Der Patient stellte sich daraufhin bei seiner Augenärztin vor, bei der er aufgrund einer schweren beidseitigen Makuladegeneration in regelmässiger Kontrolle ist. Der ophthalmologische Befund war unverändert zu den Voruntersuchungen. Auf der Notfallstation berichtete der Patient neben den Sehstörungen auch über einen diffusen «Trümmel» (Schwindelgefühl) und ein holokranielles Druckgefühl.

Das Sehvermögen des Patienten ist aufgrund der Makuladegeneration stark eingeschränkt: So liegt der Visus anamnestisch auf der rechten Seite bei 0,5, links sei der Patient fast blind. Vorerkrankungen sind ein langjähriges Asthma bronchiale und eine rechtsseitige Coxarthrose. Die Dauermedikation besteht aus Budesonid/Formoterol und Chondroitinsulfat.

\section{Status und Befunde}

Bei Eintritt lagen die Vitalparameter im Normbereich und der internistische Status war unauffällig. Der detailliert geprüfte neurologische Status war bis auf die bekannte Visuseinschränkung normal. Auch neuropsychologisch bestanden keinerlei Auffälligkeiten. Der Patient präsentierte sich bewusstseinsklar, ruhig, mit adäquater Wiedergabe der Anamnese und war sich der illusorischen Natur der Phänomene vollauf bewusst. Laboranalytisch lagen kein Entzündungszustand, keine Anämie, keine Schilddrüsenfunktionsstörung und keine Blutzucker- oder Elektrolytentgleisung vor. Auf der Notfallstation schlossen wir eine intrakranielle Blutung mittels nativer zerebraler Computertomographie aus. Die am Folgetag durchgeführte zerebrale Magnetresonanztomographie war normal. Insbesondere gab es keine Hinweise für eine frische Ischämie.

\section{Diagnose und Therapie}

Die optischen Täuschungen und visuellen Perseverationen bei einer vorbestehenden Visusminderung sind am besten durch ein Charles-Bonnet-Syndrom erklärt. Die wesentlichen Differentialdiagnosen können ausgeschlossen werden: Für eine zerebrale Ischämie im 
Bereich von Sehrinde, Hirnstamm und Thalamus lieferte die Bildgebung keine Anhaltspunkte. Die Semiologie passte nicht zu einer epileptogenen Genese (Occipitallappenanfälle präsentieren sich typischerweise mit stereotyp geformten, bewegten, meist farbigen Mustern mit anfallsweisem Auftreten für Sekunden). Der zeitliche Verlauf wie auch die visuellen Perseverationen sind schliesslich nicht typisch für eine visuelle Aura bei Migräne. Zudem war der psychopathologische Status unauffällig ohne Hinweis auf eine wahnhafte Störung.

Wir begannen einen Therapieversuch mit Quetiapin in niedriger Dosierung (12,5 mg), womit die visuellen Phänomene sistierten. Die Nebenwirkungen in Form von Sedation und Gleichgewichtsstörung waren indessen nicht akzeptabel und liessen eine Dauermedikation nicht zu. Wir wechselten deshalb auf Clonazepam (zweimal täglich 0,5 mg), worunter die Illusionen und Perseverationen nicht mehr auftraten und die Nebenwirkungen deutlich weniger stark ausgeprägt waren. Im Verlauf der folgenden vier Wochen konnte die Medikation in zwei Schritten abgesetzt werden. In der Folge nahm der Patient wieder vereinzelt Gitterlinien wahr, die er jedoch nicht mehr als störend empfand.

\section{Diskussion}

Das Charles-Bonnet-Syndrom beschreibt visuelle Täuschungen bei Patienten mit eingeschränktem Visus oder Gesichtsfeldverlust im Wachzustand und ohne zugrunde liegende psychiatrische Erkrankung [1, 2].

Charles Bonnet war ein Genfer Naturwissenschaftler, Philosoph und Anwalt im 18. Jahrhundert und beschrieb 1760 erstmalig diesen Symptomkomplex [3]. Er gilt als Entdecker der eingeschlechtlichen Fortpflanzung (Parthenogenese). Das Charles-Bonnet-Syndrom ist wahrscheinlich häufiger als allgemein angenommen, da Patienten eine psychiatrische Diagnose fürchten und die Symptome nicht rapportieren [4].

Aus pathophysiologischer Sicht besteht eine starke Assoziation des Charles-Bonnet-Syndroms mit einer Verminderung des Sehvermögens, beispielsweise bei Makuladegeneration, Glaukom, diabetischer Retinopathie und zerebrovaskulären Insulten. Fftyche et al. haben mittels funktioneller Magnetresonanztomographie gezeigt, dass verschiedene Arten visueller Illusionen mit spezifischen Arealen des Hirns korrelieren [5]. Gemäss einer verbreitet akzeptierten Theorie verursacht ein durch einen Visusverlust bedingtes Afferenzdefizit im visuellen Kortex eine gesteigerte Erregbarkeit der dortigen Neuronen [6, 7]. Eine Übererregbarkeit durch eine Deafferenzierung oder Denervierung ist dabei ein bekanntes Phänomen auch ausserhalb des visuellen
Systems [8], so zum Beispiel in Form von neuropathischen Schmerzen nach Amputationen, Nervenläsionen oder Tinnitus bei Hörverlust. Es gibt ausserdem Berichte von gesteigerter Spontanaktivität als Folge eines Afferenzverlustes. So konnten in neuronal vollständig isolierten Hirnabschnitten wie auch in vitro spontane Entladungen von Neuronen gemessen werden [6]. In wiefern pathologische Spontanaktivität beim CharlesBonnet-Syndrom tatsächlich beteiligt ist, ist nicht abschliessend geklärt. Eine andere Theorie geht davon aus, dass Störungen auf jeder Ebene des visuellen Systems zu Disinhibitionen im visuellen Kortex führen können. Die visuellen Phänomene wären somit nicht zwingend durch eine okuläre Störung verursacht [7].

Die Form der visuellen Phänomene kann äusserst vielfältig sein von statischen, simplen geometrischen Figuren bis hin zu komplexen menschenähnlichen und animierten Gestalten. Typischerweise werden sie verstärkt beim Blick auf einen weissen Hintergrund und verschwinden beim Schliessen der Augen. Der Inhalt der Illusionen wird von Patienten fast immer als unreal erkannt, dies im Gegensatz zur Halluzination (siehe auch weiter unten).

Differentialdiagnostisch sollte eine Pathologie der Netzhaut mit einer ophthalmologischen Untersuchung ausgeschlossen werden. Retinale Schäden sind oft monokular und produzieren einfache statische Phänomene, die nur wenige Sekunden andauern.

Eine Migräne-Aura kann visuelle Phänomene verursachen, die sich normalerweise auf eine Hälfte des Gesichtsfeldes beschränken, mit anderen Symptomen einer Migräne (unilaterale Kopfschmerzen, Nausea und Emesis, Phono- oder Photophobie) einhergehen und typischerweise 30 bis 60 Minuten andauern. Die Semiologie der visuellen Migräne-Auren umfasst vor allem lineare oder geometrische Muster (Zackenkranz, Fortifikationsspektren) und Flimmerskotome (Abb. 2).

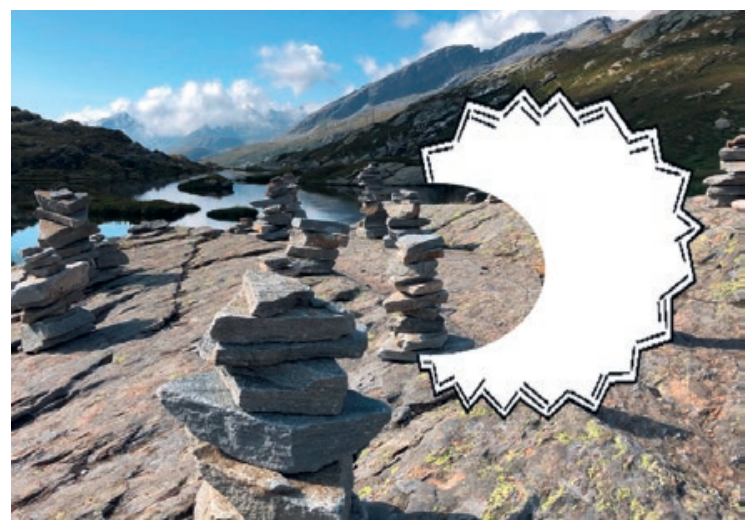

Abbildung 2: Typische visuelle Aura bei Migräne bestehend aus Fortifikationsspektrum mit zentralem Skotom (c) Rolf Erlebach). 


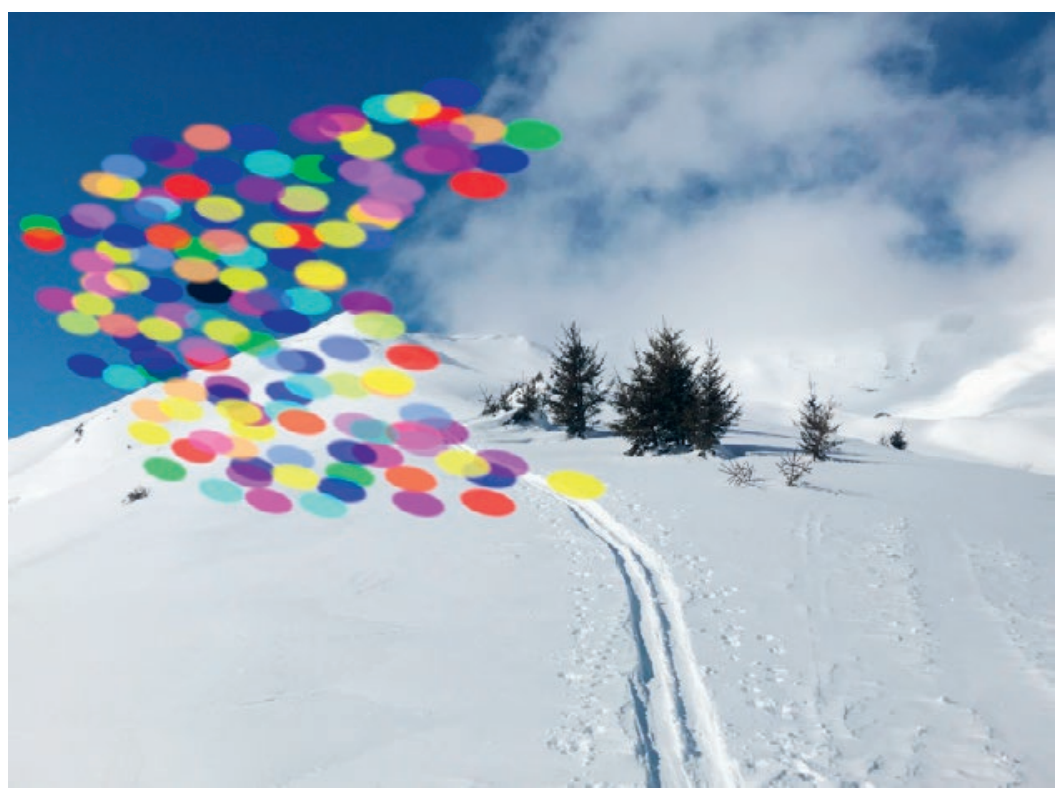

Abbildung 3: Zirkuläre farbige Figuren, wie sie bei visuellen epileptischen Halluzinationen vorkommen (ৎ Rolf Erlebach).

Epileptische visuelle Phänomene unterscheiden sich durch ein streng stereotypes Auftreten von farbigen, oft zirkulären Figuren, die sich schnell über das Gesichtsfeld bewegen und nur wenige Sekunden andauern (Abb. 3).

Unter den neurodegenerativen Erkrankungen ist es vor allem die Lewy-Body-Demenz, die früh im Krankheitsverlauf mit ausgeprägten visuellen Halluzinationen einhergeht. Typischerweise sehen die Patienten komplexe Bilder von Menschen oder Tieren, die über Sekunden bis Minuten anhalten und als real verkannt rolferlebach[at]gmail.com
CH-8610 Uster werden. Eine weitere seltene neurologische Differentialdiagnose ist die sogenannte pedunkuläre Halluzinose bei vaskulären Läsionen im Bereich des oberen Hirnstamms und des Thalamus. Diese Halluzinationen sind typischerweise besonders reich ausgestaltet: mit Figuren, Tieren, Landschaften, teilweise in verzerrten Proportionen (z.B. Gesichter). Ausserdem können sie neben visuellen auch auditorische und taktile Inhalte zeigen

Weitere Differentialdiagnosen sind akute Intoxikationen oder ein Delir, die oft mit einer quantitativen Bewusstseinsstörung einhergehen. Unter den Medikamenten sind es beispielsweise hohe Dosen von Glukokortikoiden oder Digoxin, die visuelle Halluzinationen verursachen. Psychiatrische Erkrankungen hingegen führen kaum zu isolierten visuellen Halluzinationen.

Das therapeutische Vorgehen orientiert sich an Fallserien. Es gibt geringe Evidenz für den Einsatz von Antipsychotika (z.B. Olanzapin, Quetiapin), Cholinesterase-Inhibitoren (z.B. Donepezil), Serotonin-Reuptake-Hemmern (z.B. Escitalopram) und Antiepileptika (z.B. Carbamazepin, Clonazepam, Valproat). Manche Patienten können durch Augenblinzeln oder schnelle Augenbewegungen die Illusionen unterdrücken. Aufgrund der ungenügenden Datenlage ist darum ein individuelles Vorgehen empfohlen, insbesondere da sich nicht alle Patienten durch die Illusionen gestört fühlen und sich davon distanzieren können. Die Patienten sind in der Regel beruhigt, wenn sie wissen, dass es sich nicht um eine neurodegenerative Erkrankung handelt.

\section{Disclosure statement}

Die Autoren haben keine finanziellen oder persönlichen Verbindungen im Zusammenhang mit diesem Beitrag deklariert.

\section{Literatur}

1 Menon GJ, Rahman I, Menon SJ, Dutton GN. Complex Visual Hallucinations in the Visually Impaired: The Charles Bonnet Syndrome. Surv Ophthalmol. Elsevier. 2003;48(1):58-72.

2 Kennard C. Charles Bonnet syndrome - disturbing 'playthings of the brain'. Pract Neurol. 2018;18(6):434-5.

3 Bonnet C. Essai Analytique sur les Facultés de l'Âme. 1th ed. Copenhagen: Philibert; 1760. p. 426-8.

4 Scott IU, Schein OD, Feuer WJ, Folstein MF. Visual hallucinations in patients with retinal disease. Am J Ophthalmol. 2001;131(5):590-8.

5 Ffytche DH, Howard RJ, Brammer MJ, David A, Woodruff P, Williams $S$. The anatomy of conscious vision: an fMRI study of visual hallucinations. Nat Neurosci. 1998;1(8):738-42.

6 Burke W. The neural basis of Charles Bonnet hallucinations: a hypothesis. J Neurol Neurosurg Psychiatry. 2002;73(5):535-41.

7 Pang L. Hallucinations Experienced by Visually Impaired: Charles Bonnet Syndrome. Optom Vis Sci. 2016;93(12):1466-78.

8 Møller AR. Symptoms and signs caused by neural plasticity. Neurol Res. 2001;23(6):565-72 